\title{
Diabetes mellitus and prostate cancer metabolism: Is there a relationship?
}

\author{
Hugo Pontes Antunes ${ }^{1}$, Ricardo Teixo ${ }^{2,3,5}$, João André Carvalho ${ }^{1}$, Miguel Eliseu ${ }^{1}$, Inês Marques ${ }^{2,3,5}$, \\ Ana Mamede 2, 3, 4, 5, Rita Neves 2, 3,5, Rui Oliveira 3,6, Edgar Tavares-da-Silva 1,3,5, Belmiro Parada 1,5, \\ Ana Margarida Abrantes 2, 3,5, Arnaldo Figueiredo ${ }^{1,5}$, Maria Filomena Botelho 2, 3, 5 \\ ${ }^{1}$ Urology and Transplantation Department, Coimbra Hospital and University Centre, Coimbra, Portugal; \\ ${ }^{2}$ CNC.IBILI, University of Coimbra, Coimbra, Portugal; \\ ${ }^{3}$ Institute of Biophysics, Faculty of Medicine, University of Coimbra, Coimbra, Portugal; \\ ${ }^{4}$ CICS-UBI, Health Sciences Research Centre, University of Beira Interior, Covilhã, Portugal; \\ ${ }^{5}$ Institute for Clinical and Biomedical Research (iCBR) area of Environment Genetics and Oncobiology (CIMAGO) Faculty \\ of Medicine University of Coimbra, Portugal; \\ ${ }^{6}$ Pathology Department, Coimbra Hospital and University Centre, Coimbra, Portugal
}

\begin{abstract}
Summary Objective: Our aim was to evaluate the effects of glucose levels and diabetes mellitus in prostate cancer $(\mathrm{PCa})$ biology.

Materials and methods: Two PCa cell lines (LNCap and PC3) were cultured in RPMI medium with different glucose concentrations [5mM (LG) and 25mM (HG)]. Expressions of androgen receptor, Her 2/neu and glucose transporters (GLUT1, 3, 5 and 12) were evaluated by flow cytometry. Proliferation rate was assessed by colorimetric assay MTT and cellular characterization was performed by haematoxylin and eosin staining. Additionally, we performed a cross sectional analysis of 704 patients undergoing radical prostatectomy who were divided into two groups (diabetic and non-diabetic). An analysis of clinical and histological data seeking to identify the differences on tumor aggressiveness between the two groups was performed. Results: In LNCaP cell line, when the glucose concentration in the medium increased, there was an increased in AR expression. Regarding expression of Her2/neu receptor, medium's glucose concentration significantly changed the expression of this receptor in both PC3 and LNCaP cell lines. Growth rate was higher on the HG medium for both cell lines. The clinical study of patients undergoing radical prostatectomy revealed no relationship between the presence of diabetes and the development of more aggressive tumours. Diabetic patients had significantly higher prostatic volumes, however, no significant difference was found between the relapse risk classification or the ISUP classification between the two groups.

Conclusions: Our results showed that medium glucose concentration could influence prostate cancer cells growing but not the aggressiveness.
\end{abstract}

KEY WORDS: Diabetes; Glucose; Prostate cancer; Hormonal receptors; ${ }^{18} \mathrm{~F}-\mathrm{FDG}$.

Submitted 20 July 2018; Accepted 27 July 2018

\section{INTRODUCTION}

Diabetes mellitus (DM) and prostate cancer (PCa) are two diseases that affect a large number of men in adulthood $(1,2)$. Several studies show how DM can be a risk factor for the development of several neoplasms (1-4) or even to its progression (5).
The relationship between the DM and the PCa is still not well studied, as with other neoplasms. Populational studies have shown conflicting results $(6,7)$.

The REDUCE study showed no association between the $\mathrm{DM}$ and the risk of PCa (6). On the other hand, the Prostate Cancer Prevention Trial (PCPT) shows an increased risk of PCa in diabetic patients (7). Reviews from studies of the pre-PSA era have shown a negative association between PCa and DM $(8,9)$. Latest reviews show that there may be an increased risk for high-grade tumours in diabetic patients, despite a negative association between DM and all forms of PCa (10-12).

Diabetic disease's parameters as hyperglycaemia (proven by $\mathrm{HbAlc}$ or by fasting blood glycaemia) show a linear risk association with high-grade tumours $(11,13)$. PCa diagnosis in diabetic patients may be more difficult because diabetics presents lower PSA values, lower serum testosterone and higher prostatic volumes (14).

Finally, some therapies for DM seem to have effect also on PCa, as for example metformin, which has been associated with reduction in cancer-specific mortality, as well as less recurrences after curative treatment in PCa (15). Molecular mechanisms involved in the relationship between DM and neoplasms have been the focus of several works.

The main study points were the molecular pathways associated with hyperinsulinemia $(16,17)$, insulin growth factor-1 (IGF-1) and inflammation (18). Concerning PCa and DM, scientific work at the molecular level is scarce. In addition to the pathways referred above, hyperglycaemia per se seems to have an effect on the androgen receptor (AR) expression (19).

The aim of our study was to evaluate the effects of high glucose level, one aspect of the diabetic disease, in PCa tumour biology. For this purpose, we evaluated the various molecular expression in PCa cell lines, when incubated in different glycaemic environments, simulating normal blood glucose and hyperglycaemia concentrations.

To complement the study in cell lines, we performed a revision of our patients' data subjected to radical prostate- 
ctomy and compared clinical and histological data of diabetic and non-diabetic patients.

\section{Materials AND METHOdS}

\section{Cell culture}

Prostate cancer cell lines used in this study - LNCaP (hormone-dependent) and PC3 (hormone-independent) were obtained in American Type Culture Collection (ATCC, USA). Cell lines were thawed and propagated in adherent cultures in Roswell Park Memorial Institute medium (RPMI), pH 7.4, supplemented with 10\% fetal bovine serum (Sigma F7524), 1\% antibiotic/antimycotic (Sigma A5955) and 1\% sodium pyruvate (Gibco 11360). We used two different formulations of RPMI medium: high glucose (25mM, SIGMA R4130) or low glucose (5mM).

The low glucose (LG) medium resembles normal glycaemia. To obtain $5 \mathrm{mM}$ glucose medium, we used a medium without glucose (SIGMA R1383) to which we added the appropriate amount of glucose (SIGMA G7528). Cells were maintained at $37^{\circ} \mathrm{C}$ with $5 \% \mathrm{CO}_{2}$.

\section{Expression of androgen receptor (AR) and Her2/neu}

To evaluate the expression of AR and Her2/neu, 106 cells were washed with phosphate buffered saline [PBS: $137 \mathrm{mM} \mathrm{NaCl}$ (Sigma, S7653), $2.7 \mathrm{mM} \mathrm{KCl}$ (Sigma, P9333), $10 \mathrm{mM} \mathrm{Na} 2 \mathrm{HPO} 4.2 \mathrm{H} 2 \mathrm{O}$ (Merck, 6580), 2mM KH2PO4 (Sigma, P0662), $p H=7.4$ ] by centrifugation at $209 \times \mathrm{G}$ during 5 minutes. Cells were stained with anti-AR antibody (Abcam AB9474) and with anti-Her2/neu-APC (APC, alophycoerythrin) antibody (BD BioSystems 340554) for 15 minutes at room temperature in the absence of light. After cells were washed and resuspended in $400 \mu \mathrm{L}$ of PBS. Stained cells with anti-AR were washed as described above and stained with a secondary antibody conjugated with phycoerythrin (PE, Santa Cruz Biotechnology SC3818) for 20 minutes at room temperature in the absence of light, and after were washed with PBS by centrifugation at 209xG for 5 minutes and resuspended in $400 \mu \mathrm{L}$ of PBS. The expression of AR and Her2/neu were analyzed by flow cytometry. The results obtained are expressed as mean intensity fluorescence (MIF).

\section{Proliferation rate}

To analyse PCa cells growth, 50000 cells $/ \mathrm{mL}$ were plated in 24 multiwell plates. After 24 and 96 hours, the colorimetric test MTT was performed as described elsewhere 20. The proliferation rate is given by the ratio of the absorbance measured at 96h (AD4) and measured at 24h (AD1).

\section{Cellular characterization and immunocytochemistry}

Cell lines used in this experiment were morphologically characterized using cytospin centrifugation, on a Shandon Cytospin II Cytocentrifuge at $471 \times G$ for $3 \mathrm{~min}$ and then stained using haematoxylin and eosin (H\&E). The remaining material was then centrifuged at $471 \times G$ for $5 \mathrm{~min}$. After, paraffin inclusion of the pellet was performed for immunocytochemistry, on a Ventana Marker Platform Bench Mark ULTRA IHC/ISH.
Immunocytochemical analysis was performed using formalin-fixed, paraffin-embedded sections. The avidinbiotin peroxidase complex technique was employed. Ki67 antigen (MIB-1 clone, 1:50, Ultra CC1 antigen retrival, Darko) was tested as representative of proliferation index, and evaluated by percentage of expression it was chosen for each condition a hot spot and counted the percentage of cells with nuclear expression/without expression of Ki67 in a high-power field (40x objective $0.3 \mathrm{~mm}^{2}$ ). The samples were observed under a light microscope - Nikon Eclipse 50i, and images were obtained using a Nikon-Digital Sight DS-Fil camera.

\section{Glucose transporters (GLUTs) quantification}

To evaluate the membrane expression of glucose transporters previously referred, 106 cells were washed by centrifugation with $\mathrm{PBS}$ at $209 \times \mathrm{G}$ during 5 minutes. Cells were stained with anti-GLUT1-PE (RED Systems FAB1418P), anti-GLUT3 (RED Systems MAB1415), antiGLUT5 (RED Systems MAB1349) and anti-GLUT12 (Santa Cruz Biotechnology sc-161659) for 15 minutes at room temperature and in the absence of light. After, cells stained with monoclonal antibody anti-GLUT1-PE were washed with PBS by centrifugation at $209 \times \mathrm{G}$ during 5 minutes and resuspended in $400 \mu \mathrm{L}$ of PBS. Stained cells with the antibodies anti-GLUT3, anti-GLUT5 and antiGLUT12 were washed with PBS by centrifugation at 209xG for 5 minutes and stained with a secondary antibody conjugated with PE (Santa Cruz Biotechnology sc3818) for 20 minutes at room temperature in the absence of light. After incubation, cells were washed with PBS by centrifugation at $209 \times \mathrm{G}$ during 5 minutes and resuspended in $400 \mu \mathrm{L}$ of PBS. The expression of GLUTs was analysed by flow cytometry. The results obtained are expressed as MIF.

\section{${ }^{18} \mathrm{~F}$-Fluorodeoxyglucose $\left({ }^{18} \mathrm{~F}\right.$-FDG) uptake studies} A cell suspension of $2 \times 106$ cells $/ \mathrm{mL}$ was prepared to perform the uptake studies. Obtained the suspension, it was left to rest at $37^{\circ} \mathrm{C}$ in $25 \mathrm{~cm}^{2}$ flasks. After 60 minutes, $25 \mu \mathrm{Ci} / \mathrm{mL}$ of ${ }^{18} \mathrm{~F}-\mathrm{FDG}$ was added to cell suspension.

At 5, 30, 60, 90 and 120 minutes, $200 \mu \mathrm{L}$ of cell suspension were collected for microtubes with iced PBS. The samples were centrifuged at $209 \times G$ for 1 minute to separate the pellet from the supernatant, having this been collected to an identified tube. After the separation of pellets and supernatants, the ${ }^{18} F-F D G$ uptake was determined by counting both fractions in a well counter (CAPINTEC CRC-15W) in counts per minute (CPM). The uptake studies were performed in normoxia $\left(95 \% \mathrm{O}_{2}\right.$ and $\left.5 \% \mathrm{CO}_{2}\right)$, as well as in hypoxia $\left(93 \% \mathrm{~N}_{2}, 2 \% \mathrm{O}_{2}\right.$ and $\left.5 \% \mathrm{CO}_{2}\right)$ conditions. Studies in hypoxia were performed in a controlled environment chamber (PlasLabs Lamsing, Mich. 800-866-7527).

\section{Clinical study}

A cross sectional analysis of all patients undergoing radical prostatectomy between January 2009 and December 2016 in urology department was performed. We evaluated 704 patients who were divided into two groups (diabetic and non-diabetic). We assessed clinical and histological parameters such as age at PCa diagnosis, pre- 
operative PSA, diagnostic biopsy results and surgical specimen pathology. All patients classified as diabetic were already diagnosed prior to radical prostatectomy. The diagnosis was in all cases performed by endocrinologists or general practitioners, with all patients being followed up on a specific diabetes mellitus consultation. All patients underwent open radical prostatectomy.

\section{Statistical analysis}

Statistical analysis was performed using the $I B M^{\circledR}$ SPSS ${ }^{\circledR}$ software v. 22.0 (IBM Corporation, Armonk, New York, USA). The normality distribution of the variables was confirmed through Shapiro-Wilk. Student t-test (parametric) was used in case of normal distribution and homogeneity of variance, otherwise, Mann-Whitney test (nonparametric) was used. In order to compare cell lines, one-factor analysis of variance (ANOVA) parametric test was used in case of normal distribution and homogeneous variances of the variables, otherwise KruskalWallis nonparametric test was used. Multiple comparisons were obtained after Games-Howell correction. The Chi-Square test was used to analyse categorical variables. A significant level of 5\% was adopted for all comparisons.

\section{RESULTS}

\section{Expression of AR and Her2/neu}

To characterize both PC3 and LNCaP cell lines we evaluated hormonal androgen receptor and Her2/neu with high and low glucose concentration medium. Results are presented in Figure 1.

According to Figure 1A, PC3 cell line does not express androgen receptor in both conditions, as for this receptor, we only consider a positive staining when MIF values are higher than 10. On the other hand, LNCaP cell line (Figure 1B) expresses this receptor in both culture conditions but show a significantly higher expression of AR when cultured in high glucose concentration medium ( $<<0.05$ ). Regarding expression of Her2/neu receptor in prostate cancer cell lines, it is possible to observe that glucose concentration in the medium significantly alter the expression of this receptor in both PC3 (Figure 1C, $\mathrm{p}=0.036$ ) and LNCaP (Figure 1D, p < 0.001) cell lines, with an increased expression in high glucose medium. Our results also highlight that androgen-dependent cell line LNCaP presents a higher expression of this receptor compared to androgen-independent cell line PC3, both in $H G(p<0.001)$ and in $L G$ $(\mathrm{p}<0.01)$ medium.

\section{Proliferation rate}

To evaluate the proliferation rate of $\mathrm{PCa}$ cell lines, the ratio AD4/AD1 was calculated and is represented in Figure 2.

\section{Figure 1.}

Results showed that PC3 cell line has a higher growth rate than LNCaP in both media considered in our work. PC3 cell line presents an AD4/ADl ratio of 9.2 when cultured in high glucose condition and an AD4/AD1 ratio of 6.1 when cultured in low glucose. Thus, it presents a significantly higher proliferative rate in high glucose conditions $(p<0.001)$. The same observation is valid for LNCaP cells, presenting an AD4/ADl ratio of 3.1 when cultured in high glucose condition a significant different value when compared with the AD4/ADl ratio of 1.5 when cultured in low glucose conditions $(\mathrm{p}=0.003)$. We can also observe that proliferative rate is significantly higher in PC3 cells than in LNCaP cells, in both glucose conditions, high $(\mathrm{p}<0.001)$ and low $(\mathrm{p}<0.001)$.

\section{Cellular characterization and immunocytochemistry}

Percentage of positive Ki67 cell of prostate cancer cell lines in high and low glucose are, respectively, 38.7\% and $9.9 \%$ in PC3 cell lines and $11.2 \%$ and $3.6 \%$ in LNCaP cell lines. The percentage of Ki67 positive cells is significantly higher in cells cultured in high glucose medium, in both LNCaP and PC3 cell lines. Moreover, we observed a higher expression of Ki67 in the PC3 cell line in both culture media.

\section{Glucose transporters (GLUTs) quantification}

Glucose transporters expression is resumed in Figure 3. Our results showed that there are differences in the expression of glucose transporters between the two cell lines under study. LNCaP cell line presented a higher expression of GLUT12 than PC3 cells when cultured in low glucose condition $(\mathrm{p}=0.013)$.

Expression of androgen receptor in PC3 $(A)$ and $L N C a P(B)$ in high glucose $(H G)$ and low glucose (LG). Expression of her2/neu receptor in PC3 (C) and LNCaP (D) in high glucose (HG) and low glucose (LG), expressed as mean intensity fluorescence (MIF). For each condition, results were obtained with a minimum of eight experiments. Graphs represent mean \pm standard error.

Statistical significance: ${ }^{\star} p<0.05 ; * \star \star p<0.001$.

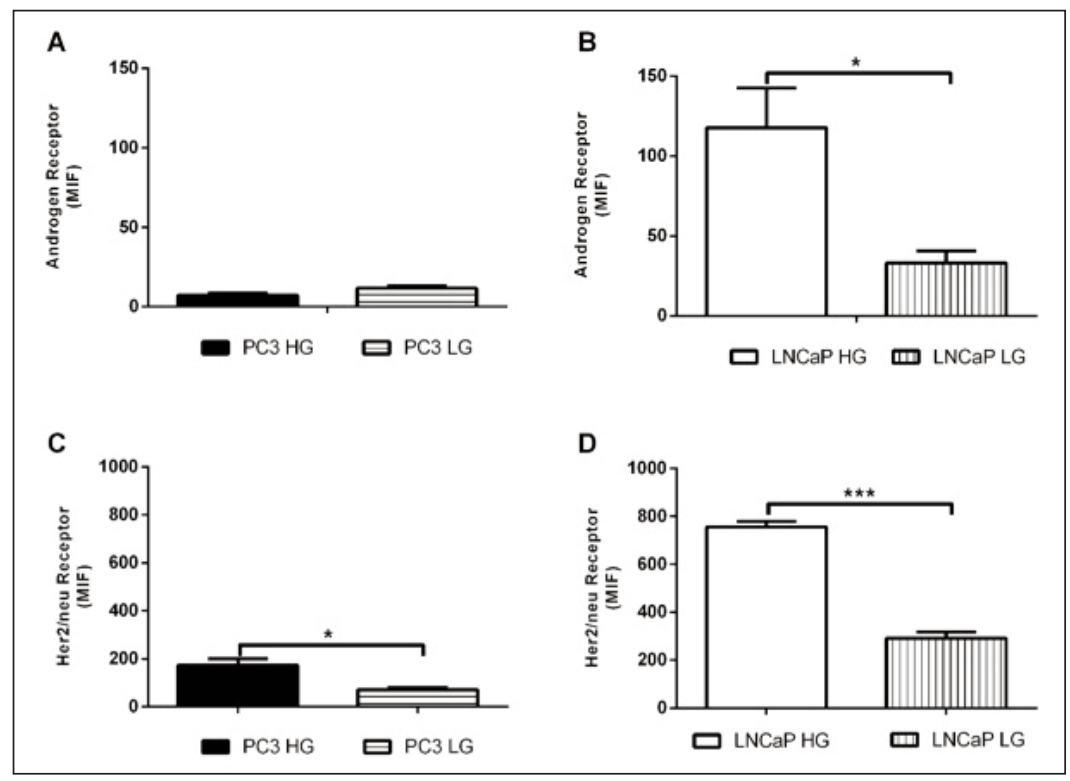


Figure 2.

Results represent the ratio between the measured absorbance after 96h (day 4, $A D 4$ ) and after 24h (day 1, AD1) for PC3 (A) and LNCaP (B). Results were obtained with six independent experiments. Values represent mean \pm standard error Statistical significance: $* \star p<0.01 ; * \star \star p<0.001$.

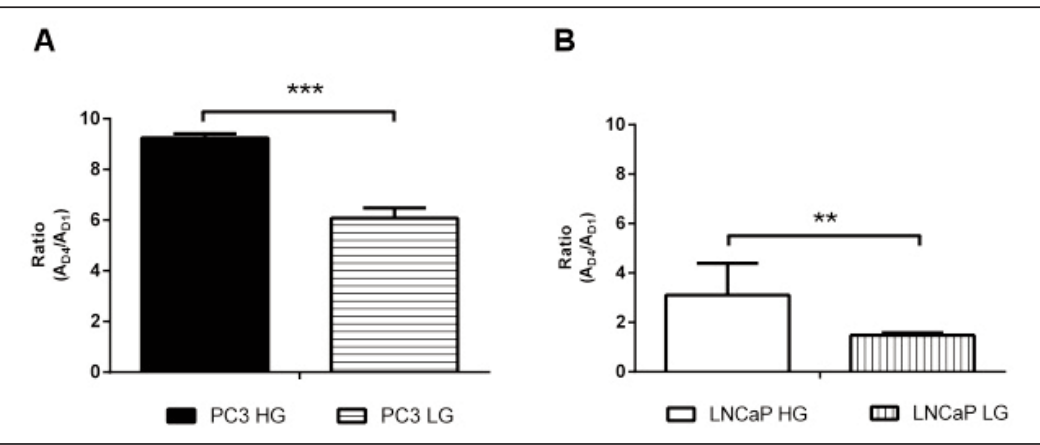

Figure 3.

Expression of GLUTS in prostate cancer cell line $L N C a P(A)$ and $P C 3(B)$ in high and low glucose media presented as mean intensity fluorescence. Results are expressed as mean \pm standard error of a minimum of three independent experiments Statistical significance: ${ }^{*} p<0.01$.
A

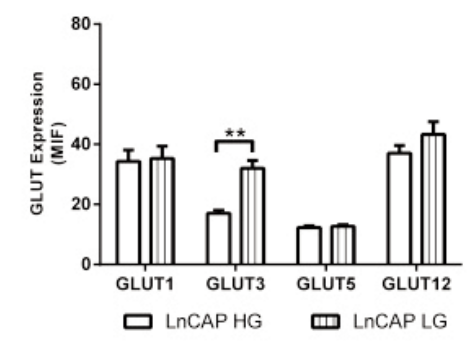

B

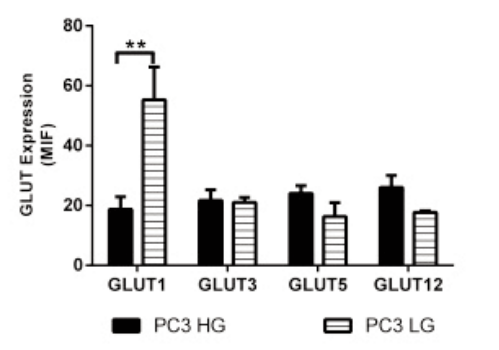

Figure 4.

${ }^{18}$ F-FDG uptake in PC3 (A) and LNCaP (B) prostate cancer cells cultures in high and low glucose media, expressed as percentage of uptake. Results are presented as mean \pm standard error of a minimum of four independent experiments Statistical significance: ${ }^{\star} p<0.05 ;{ }^{\star}{ }_{p}<0.01 ;{ }^{\star *} p<<0.001$.

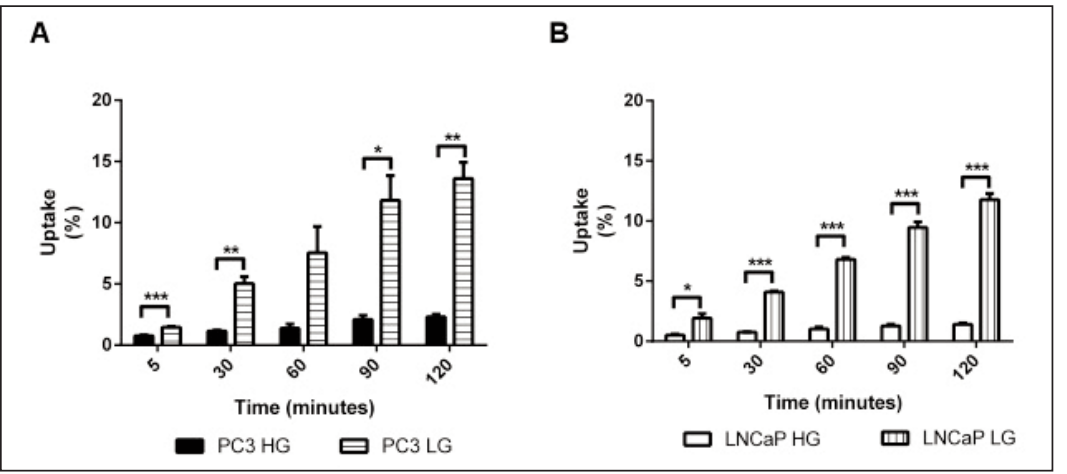

${ }^{18}$ F-Fluorodeoxyglucose $\left({ }^{18} \mathrm{~F}-\mathrm{FDG}\right)$ uptake studies

In our work the uptake profile of ${ }^{18} \mathrm{~F}$ FDG was determined in prostate cancer cell lines when cultured in high and low glucose media. Our results (Figure 4) showed that there are no alterations in ${ }^{18}$ F-FDG uptake over time when PC3 and LNCaP cell lines are cultured in high glucose medium. Moreover, when cells are culture in low glucose concentration, ${ }^{18}$ F-FDG uptake significantly increases with time when compared with cells cultured in high glucose concentration. On PC3 cell line, we observed a significantly higher uptake on cells culture on LG compared with HG after 5 minutes $(1.46 \% \pm 0.06 \%$ vs. $0.76 \% \pm 0.07 \%, \mathrm{p}<0.001), 30$ minutes $(5.02 \% \pm 0.58 \%$ vs. $1.15 \% \pm 0.07 \%, p=$ $0.010), 90$ minutes $(11.83 \% \pm 2.01 \%$ vs. $2.09 \% \pm 0.33 \%, p=0.026)$ and 120 minutes $(13.60 \% \pm 1.33 \%$ vs. $2.31 \% \pm$ $0.22 \%, p=0.005)$.

On LNCaP cells, we observed a significantly higher uptake on cells culture on LG compared with HG after 5 minutes $(1.92 \% \pm 0.37 \%$ vs. $0.51 \% \pm 0.09 \%$, $\mathrm{p}<0.045), 30$ minutes $(4.10 \% \pm$ $0.10 \%$ vs. $0.76 \% \pm 0.02 \%, p<0.001)$, 60 minutes $(6.80 \% \pm 0.18 \%$ vs. $1.02 \%$ $\pm 0.17 \%, \mathrm{p}<0.001), 90$ minutes $(9.46 \% \pm 2.01 \%$ vs. $1.28 \% \pm 0.14 \%$, $\mathrm{p}<0.001)$ and 120 minutes $(11.78 \% \pm$ $0.51 \%$ vs. $1.41 \% \pm 0.12 \%, \mathrm{p}<0.001)$.

\section{Clinical study}

Demographic and clinicopathological data of patients undergoing radical prostatectomy are shown in Table 1 . The rate of diabetic patients was $21.2 \%$. Diabetic patients had higher prostatic volumes. However, the diagnosis of DM did not show relationship with the development of tumours with more aggressive histology or staging.

\section{Discussion}

As mentioned before, the correlation between DM and some neoplasms, like lung, colorectal or breast cancer, is already known (1-3), however, the relationship with PCa was not established

We also observed that GLUT1, GLUT3 and GLUT5 expression is not different between the two prostate cancer cell lines.

Moreover, in LNCaP cells, an increased expression of GLUT3 were observed when cells were cultured in low glucose medium $(p=0.016)$. In PC3 cell line, this differential expression due to culture media is observed only for GLUT1 ( $p=0.013)$. yet. Similarly, populational studies are not clear on establishing an association between this two diseases (68). The diabetic disease has several aspects capable of exerting influence on PCa, like hyperinsulinemia (16), IGF-1, inflammation and also its treatment (18).

In our work, we evaluated another aspect of DM, the hyperglycaemia, and its action on PCa behaviour at a molecular level, by using an in vitro model of two PCa 
Table 1.

Demographic and clinicopathological features of patients undergoing radical prostatectomy.

\begin{tabular}{|c|c|c|c|}
\hline & $\begin{array}{l}\text { Diabetic group } \\
(\mathrm{n}=149)\end{array}$ & $\begin{array}{l}\text { Non-diabetic group } \\
\quad(n=555)\end{array}$ & $P$ value \\
\hline Age (years), mean \pm SD & $64.5 \pm 5.6$ & $62.8 \pm 6.7$ & 0.003 \\
\hline PSA preoperative $(\mathrm{ng} / \mathrm{mL})$, mean \pm SD & $9.2 \pm 6.3$ & $9.9 \pm 12.0$ & NS \\
\hline Preop. fasting glycaemia (mg/dL), mean \pm SD & $114.0 \pm 25.4$ & $93.9 \pm 12.6$ & 0.001 \\
\hline Prostatic volume (cc), mean \pm SD & $54.2 \pm 21.3$ & $47.7 \pm 18.1$ & 0.001 \\
\hline Risk groups, $\mathrm{n}(\%)$ & & & NS \\
\hline Low-risk & $50(33.6 \%)$ & $208(37.5 \%)$ & \\
\hline Intermediate-risk & $80(53.5 \%)$ & $261(47.0 \%)$ & \\
\hline High-risk & $17(11.4 \%)$ & $63(11.4 \%)$ & \\
\hline ISUP of RP specimen, $\mathbf{n}(\%)$ & & & NS \\
\hline ISUP Grade 1 & $31(20.8 \%)$ & $118(21.2 \%)$ & \\
\hline ISUP Grade 2 & $96(64.4 \%)$ & $364(65.6 \%)$ & \\
\hline ISUP Grade 3 & $13(8.7 \%)$ & 54 (9.7\%) & \\
\hline ISUP Grade 4 & $3(2.1 \%)$ & $12(2.2 \%)$ & \\
\hline ISUP Grade 5 & $6(4.0 \%)$ & $7(1.3 \%)$ & \\
\hline Perineural invasion, $\mathbf{n}(\%)$ & $116(83.5 \%)$ & $456(86.4 \%)$ & NS \\
\hline TNM staging, $n(\%)$ & & & NS \\
\hline pT2 & $85(57.0 \%)$ & $328(59.1 \%)$ & \\
\hline pT3 & 64 (43.0\%) & 227 (40.9\%) & \\
\hline pNO & 93 (62.4\%) & 357 (64.3\%) & \\
\hline pN1 & $12(8.1 \%)$ & $37(6.7 \%)$ & \\
\hline $\mathrm{pNx}$ & $44(29.5 \%)$ & $161(29.0 \%)$ & \\
\hline Positive margins, $\mathbf{n}(\%)$ & $41(27.5 \%)$ & $138(24.9 \%)$ & NS \\
\hline
\end{tabular}

cell lines incubated with different glucose concentrations, resembling a normal glycaemia (LG) and hyperglycaemia (HG).

Our results showed that PC3 cell line do not significantly express AR, while LNCaP cell line have a higher expression of this receptor, as was proved by Tilley (21) in 1990. For LNCaP cell line we observed that when medium's glucose concentration increased, there was an increased in AR expression. This may be explained, in part, by the AR role on the glucose uptake and glycolysis regulation (22). It seems that AR stimulates glycolysis via the metabolic sensor $5^{\prime}$-AMP activated protein kinase (AMPK) not only for adenosine 5'-triphosphate (ATP) production $(23,24)$ but also for de novo lipid synthesis (25). This is consistent with the typical Warburg effect for cancer cells metabolism (26), which is an inefficient way to generate ATP in order to acquire nutrients (26). This effect appears to take place in prostate cancer under the AR regulation (22).

Her2/neu has been indicated as a factor of cancer aggressiveness in other types of cancer (27) and in PCa was also related with AR activation in castration resistant prostate cancer (28). Our results showed that Her2/neu receptor expression was also significantly different with both media glucose concentrations in the two cell lines. There was an increased Her2/neu receptor expression with the HG medium. As far as we know, this association was not studied until today in prostate cancer cells, but in breast cancer cells was identified a relationship between Her2/neu and the glycolytic phenotype (29). It appears that Her2/neu overexpression increases activation of Akt, which leads to an up-regulation of GLUT1 and a down-regulation of M2PK (M2 pyruvate kinase isoenzyme) (29). This down regulation of M2PK leads to an accumulation of glycolytic metabolites, providing substrates for synthetic processes (29). Thus, as we mentioned before, PCa cells also stimulates glycolysis for a synthetic purpose (25), may the process seen in breast cancer cells also happens with them. ${ }^{18} \mathrm{~F}-\mathrm{FDG}$ is an analogous of glucose radiolabelled with Fluor-18. In the uptake studies we verified that the uptake of the ${ }^{18} \mathrm{~F}-\mathrm{FDG}$ glucose analogous depends of the medium composition. Besides this it is important to have in account the uptake mechanism in which ${ }^{18} F-F D G$ enters in the cell by facilitated diffusion mediated by membrane glucose transporters, and after it is phosphorylated by hexokinase to FDG-6-phosphate. Further the phosphorylation concerning the very strict structural and geometric demands of the reaction, the phosphoglucose isomerase does not recognize FDG-6phosphate and consequently there will be accumulation on the cytoplasm, which means that ${ }^{18} \mathrm{~F}-\mathrm{FD}$-6-6-P remains inside the cell capable of being detected during the time that remains radioactive. The distribution of radiolabelled FDG reflects not the glycolytic pathway but the exaggerated need of glucose according to the Warburg effect (30). The ${ }^{18}$ F-FDG uptake by the cells was higher with low glucose concentration medium. This can be explained by the absence of competitor in the transporter, which is the glucose present in the medium. Since PCa cells are metabolically very active, they are greedy to glucose and the lower the content of glucose, the higher is the ${ }^{18} \mathrm{~F}$-FDG uptake. This has clinical relevance because the better the glucose control is, the better will be the images of the PET scan with ${ }^{18} \mathrm{~F}$-FDG. As we determined by the proliferation rate, we verified interesting results, as the PC3 cell line is the one who present a higher growth rate and for both cell lines we saw that the HG medium had the shorter doubling time. Moreover, the fact that cells cultured in LG presented a higher ${ }^{18} \mathrm{~F}-\mathrm{FDG}$ uptake even having a lower proliferation rate highlights the clinical relevance of a good glucose control when a PET scan with ${ }^{18} \mathrm{~F}-\mathrm{FDG}$ is performed.

GLUT proteins facilitate glucose transport across biological membranes. Different expressions of GLUTs have been related with different kinds of cancer and with cancer specific survival (31-35). GLUTs 1,3 and 12 are known for being expressed in PCa (31). In our work we observed a similar expression of the investigated glucose transporters in both culture media, exception for GLUT1 in PC3 cells and GLUT3 in LNCaP cells. With the LG medium we observed a significant increase GLUT1 expression in the PC3 cells and an increase GLTU3 expression in the LNCaP cells. Also, in LNCaP cells, there is a higher expression of GLUT1, GLUT3 and GLUT12 compared with PC 3 cells when both cell lines are cultured in HG conditions, showing that maybe these 
transporters could be related with the AR (24). Despite the results in cell lines, our clinical sample did not show significant differences between tumour development in diabetic and non-diabetic patients. Diabetic patients had higher prostatic volumes. In terms of oncologic staging we did not find significant differences, however, we found that the rate of patients with grade 5 of the ISUP classification is higher in diabetic patients $(4.0 \%$ vs. $1.3 \%$ ), although this difference did not reach statistical significance. This result is in agreement with other studies that have reported that DM mainly decreases the risk of low-grade tumors and, to a lesser degree, the risk of high-grade tumors. Consequently, it is plausible to assume that DM changes the proportions of PCa grades favoring high-grade tumors $(7,11)$. Some series of patients undergoing RP have shown that DM is associated with a higher PCa grade (36). However, a previous analysis of the CaPSURE database found no association between DM and PCa aggressiveness (37). Thus, more studies in this area will be necessary to really clarify the relationship between DM and PCa aggressiveness.

\section{Conclusions}

Our results showed that glucose levels could influence prostate cancer cells behaviour. In the HG medium PCa cells had a more aggressive pattern, with higher expression of AR (only for LNCaP) and Her2/neu (for both cell lines) and a higher proliferation rate demonstrated by Ki67 staining and ratio AD4/AD1. GLUT expression had slight variations in both culture conditions, but its expression is significantly higher in LNCaP cells. Thus, incubation of cells in hyperglycaemia-like conditions induced a more aggressive molecular phenotype. This could be one of the link aspects between DM and PCa.

\section{REFERENCES}

1. Lee J-Y, Jeon I, Lee JM, et al. Diabetes mellitus as an independent risk factor for lung cancer: a meta-analysis of observational studies. Eur J Cancer. 2013; 49:2411-2423.

2. Yoon JM, Son KY, Eom CS, et al. Pre-existing diabetes mellitus increases the risk of gastric cancer: a meta-analysis. World J Gastroenterol. 2013; 19:936-945.

3. Luo S, Li J-Y, Zhao L-N, et al. Diabetes mellitus increases the risk of colorectal neoplasia: An updated meta-analysis. Clin Res Hepatol Gastroenterol. 2016; 40:110-23.

4. Liaw Y-P, Ko P-C, Jan S-R, et al. Implications of Type1/2 Diabetes Mellitus in Breast Cancer Development: A General Female Population-based Cohort Study. J Cancer. 2015; 6:734-739.

5. Ryu TY, Park J, Scherer PE. Hyperglycemia as a risk factor for cancer progression. Diabetes Metab J. 2014; 38:330-336.

6. Wu C, Moreira DM, Gerber L, et al. Diabetes and prostate cancer risk in the REDUCE trial. Prostate Cancer Prostatic Dis. 2011; 14:326-331.

7. Gong Z, Neuhouser ML, Goodman PJ, et al. Obesity, diabetes, and risk of prostate cancer: results from the prostate cancer prevention trial. Cancer Epidemiol Biomarkers Prev. 2006; 15:1977-1983.

8. Kasper JS, Giovannucci E. A meta-analysis of diabetes mellitus and the risk of prostate cancer. Cancer Epidemiol Biomarkers Prev. 2006; 15:2056-2062
9. Bonovas S, Filioussi K, Tsantes A. Diabetes mellitus and risk of prostate cancer: a meta-analysis. Diabetologia. 2004; 47:1071-1078.

10. Leitzmann MF, Ahn J, Albanes D, et al. Diabetes mellitus and prostate cancer risk in the Prostate, Lung, Colorectal, and Ovarian Cancer Screening Trial. Cancer Causes Control. 2008; 19:1267-1276.

11. Park J, Cho SY, Lee YJ, et al. Poor glycemic control of diabetes mellitus is associated with higher risk of prostate cancer detection in a biopsy population. PLoS One 2014;9:e104789.

12. Cai H, Xu Z, Xu T, et al. Diabetes mellitus is associated with elevated risk of mortality amongst patients with prostate cancer: a meta-analysis of 11 cohort studies. Diabetes Metab Res Rev. 2015; 31:336-43.

13. Park SM, Lim MK, Shin SA, Yun YH. Impact of prediagnosis smoking, alcohol, obesity, and insulin resistance on survival in male cancer patients: National Health Insurance Corporation Study. J Clin Oncol. 2006; 24:5017-5024.

14. Pierce BL. Why are diabetics at reduced risk for prostate cancer? A review of the epidemiologic evidence. Urol Oncol. 2012; $30: 735-743$

15. Hwang IC, Park SM, Shin D, et al. Metformin association with lower prostate cancer recurrence in type 2 diabetes: a systematic review and meta-analysis. Asian Pac J Cancer Prev. 2015; 16:595600 .

16. Richardson LC, Pollack LA. Therapy insight: Influence of type 2 diabetes on the development, treatment and outcomes of cancer. Nat Clin Pract Oncol. 2005; 2:48-53.

17. Argilés JM, López-Soriano FJ. Insulin and cancer. Int J Oncol. 2001; 18:683-687.

18. Joung KH, Jeong J-W, Ku BJ. The association between type 2 diabetes mellitus and women cancer: the epidemiological evidences and putative mechanisms. Biomed Res Int. 2015; 2015:920618.

19. Barbosa-Desongles A, Hernández C, De Torres I, et al. Diabetes protects from prostate cancer by downregulating androgen receptor: new insights from LNCaP cells and PAC120 mouse model. PLoS One. 2013; 8:e74179.

20. Mamede AC, Abrantes AM, Pedrosa L, et al. Beyond the limits of oxygen: effects of hypoxia in a hormone-independent prostate cancer cell line. ISRN Oncol. 2013; 2013:918207.

21. Tilley WD, Wilson CM, Marcelli M, McPhaul MJ. Androgen receptor gene expression in human prostate carcinoma cell lines. Cancer Res. 1990; 50:5382-5386.

22. Massie CE, Lynch A, Ramos-Montoya A, et al. The androgen receptor fuels prostate cancer by regulating central metabolism and biosynthesis. EMBO J. 2011; 30:2719-2733.

23. Tennakoon JB, Shi Y, Han JJ, et al. Androgens regulate prostate cancer cell growth via an AMPK-PGC-1 $\alpha$-mediated metabolic switch. Oncogene. 2014; 33:5251-5261.

24. Vaz CV, Marques R, Alves MG, et al. Androgens enhance the glycolytic metabolism and lactate export in prostate cancer cells by modulating the expression of GLUT1, GLUT3, PFK, LDH and MCT4 genes. J Cancer Res Clin Oncol. 2016; 142:5-16.

25. Moon J-S, Jin W-J, Kwak J-H, et al. Androgen stimulates glycolysis for de novo lipid synthesis by increasing the activities of hexokinase 2 and 6-phosphofructo-2-kinaselfructose-2,6-bisphosphatase 2 in prostate cancer cells. Biochem J. 2011; 433:225-233.

26. Vander Heiden MG, Cantley LC, Thompson CB. Understanding the Warburg effect: the metabolic requirements of cell proliferation. Science. 2009; 324:1029-1033. 
27. Jensen BV, Johansen JS, Price PA. High levels of serum HER2/neu and YKL-40 independently reflect aggressiveness of metastatic breast cancer. Clin Cancer Res. 2003; 9:4423-4434.

28. Guyader C, Céraline J, Gravier E, et al. Risk of hormone escape in a human prostate cancer model depends on therapy modalities and can be reduced by tyrosine kinase inhibitors. PLoS One. 2012; 7:e42252.

29. Schmidt M, Voelker H-U, Kapp M, et al. Glycolytic phenotype in breast cancer: activation of Akt, up-regulation of GLUT1, TKTL1 and down-regulation of M2PK. J Cancer Res Clin Oncol. 2010; 136:219225.

30. Jadvar H. Imaging evaluation of prostate cancer with 18F-fluorodeoxyglucose PET/CT: utility and limitations. Eur J Nucl Med Mol Imaging. 2013; 40 Suppl 1:S5-10.

31. Barron CC, Bilan PJ, Tsakiridis T, Tsiani E. Facilitative glucose transporters: Implications for cancer detection, prognosis and treatment. Metabolism. 2016; 65:124-139.

32. Godoy A, Ulloa V, Rodríguez F, et al. Differential subcellular distribution of glucose transporters GLUT1-6 and GLUT9 in human cancer: ultrastructural localization of GLUT1 and GLUT5 in breast tumor tissues. J Cell Physiol. 2006; 207:614-627.

\section{Correspondence}

Hugo Manuel Pontes Antunes, MD (Corresponding Author)

hugoantunes4@gmail.com

Urology and Transplantation Dpt, Coimbra Hospital and University Centre

Rua Padre Sebastião, nº12 - 2C, 3040-376 Coimbra, Portugal

Ricardo Teixo, MD

rcardoteixo@gmail.com

João André Carvalho, MD

jccarvalho@gmail.com

Miguel Eliseu, MD

migeliseu3@gmail.com

Inês Marques, $M D$

Ana Mamede,MD

Rita Neves, MD

Rui Oliveira, MD

Edgar Tavares-da-Silva, MD

Belmiro Parada, MD

Ana Margarida Abrantes, MD

Arnaldo Figueiredo, MD

Maria Filomena Botelho, MD
33. Sakashita M, Aoyama N, Minami R, et al. Glut1 expression in $\mathrm{T} 1$ and T2 stage colorectal carcinomas: its relationship to clinicopathological features. Eur J Cancer. 2001; 37:204-209.

34. Kawamura T, Kusakabe T, Sugino T, et al. Expression of glucose transporter-1 in human gastric carcinoma: association with tumor aggressiveness, metastasis, and patient survival. Cancer. 2001; 92:634-641.

35. Brito AF, Abrantes AM, Ribeiro $M$, et al. Fluorine-18 Fluorodeoxyglucose Uptake in Hepatocellular Carcinoma: Correlation with Glucose Transporters and p53 Expression. J Clin Exp Hepatol. 2015; 5:183-189.

36. Jayachandran J, Aronson WJ, Terris MK, et al. Diabetes and outcomes after radical prostatectomy: Are results affected by obesity and race? Results from the shared equal-access regional cancer hospital database. Cancer Epidemiol Biomarkers Prev. 2010; 19:9-17.

37. Chan JM, Latini DM, Cowan J, et al. History of Diabetes, Clinical Features of Prostate Cancer, and Prostate Cancer Recurrence-Data from CaPSURE(TM) (United States). Cancer Causes Control. 2005; 16:789-797. 